\title{
Rationalizable Solutions to Pure Population Problems*
}

\author{
by \\ Charles Blackorby, Walter Bossert, and David Donaldson
}

September 1997

Charles Blackorby: University of British Columbia and GREQAM

Walter Bossert: University of Nottingham

David Donaldson: University of British Columbia

* Financial support through a grant from the Social Sciences and Humanities Research Council of Canada is gratefully acknowledged. 


\begin{abstract}
In pure population problems, a single resource is to be distributed equally among the agents in a society, and the social planner chooses population size(s) and per-capita consumption(s) for each resource constraint and set of feasible population sizes within the domain of the solution. This paper shows that a weak condition regarding the possible choice of a zero population is necessary and sufficient for the rationalizability of a solution by a welfarist social ordering. In addition, solutions that are rationalized by critical-level generalized utilitarianism are characterized by means of a homogeneity property.

Journal of Economic Literature Classification Number: D63.
\end{abstract}

Keywords: Pure Population Problems, Social Choice, Rationalizability. 


\section{Introduction}

Pure population problems can be used to assess the performance of population principles. ${ }^{1}$ In the pure population problem considered in this paper, a given amount of a single resource is to be distributed equally among the individuals who are alive. The policy decision to be made is the choice of population size and a level of per-capita consumption. An equal distribution of consumption is assumed in order to focus on the population issue; this is plausible if individuals have identical concave utility functions.

A life, taken as a whole, is worth living if lifetime utility is above neutrality. The standard normalization in the population-ethics literature is a utility level of zero to represent a neutral life. ${ }^{2}$ Given this normalization, the following solutions to pure population problems have been proposed and discussed in the literature.

In the discrete case, the average-utilitarian solution ${ }^{3}$ recommends an optimal population size of one, regardless of the amount of the resource available. ${ }^{4}$ If population size is a continuous variable, the average-utilitarian solution does not exist.

The classical-utilitarian solution selects the population size that maximizes the sum of differences between the utilities of those alive and the utility level representing a neutral life. Given the zero normalization for neutrality, classical utilitarianism can be rationalized by a value function which is equal to total utility. ${ }^{5}$ Unlike average utilitarianism, classical utilitarianism selects optimal population sizes that increase as the amount of the resource increases. Furthermore, classical utilitarianism produces a well-defined solution regardless of whether population size is treated as a continuous or a discrete variable.

The class of critical-level utilitarian solutions generalizes classical utilitarianism. According to the rationalization of a critical-level utilitarian solution, states of the world are ranked by comparing the sums of the differences between individual utility levels and a fixed critical level of lifetime utility. If the critical level is equal to zero (neutrality), classical utilitarianism results. We recommend the choice of a critical level above neutrality in order to avoid the repugnant conclusion (Parfit [1976, 1982, 1984]). A population principle leads to the repugnant conclusion if, for any state of the world where everyone alive experiences an arbitrarily high level of well-being, there exists a better state with a sufficiently large population where each person's utility is above but arbitrarily close to

\footnotetext{
1 See Blackorby and Donaldson $[1979,1984]$.

2 See Broome [1993] for a discussion of neutrality and its normalization to zero.

3 See, for instance, Blackorby and Donaldson [1984], Bossert [1990a,b], and Hurka [1982] for discussions of average utilitarianism and its generalizations.

4 Average utilitarianism can be amended to allow for the possibility of an optimal population size of zero if the amount of the resource becomes very low.

5 Dasgupta [1993, 1994] uses a negative utility level to represent neutrality. In that case, total utility is not the value function for classical utilitarianism.
} 
neutrality. Classical utilitarianism leads to the repugnant conclusion, but average utilitarianism and critical-level utilitarianism with a positive critical level do not. If critical-level utilitarianism is applied to the pure population problem, the optimal population size is lower for higher values of the critical level. ${ }^{6}$ Again, the critical-level utilitarian solutions are well-defined for both discrete and continuous population sizes. See Blackorby, Bossert, and Donaldson [1995b, 1996, 1997] and Blackorby and Donaldson [1984] for discussions of critical-level utilitarianism.

All of the above rationalizations can be generalized by applying a continuous, increasing transformation to the individual utilities. This allows for the possibility of expressing inequality aversion in utilities. For example, critical-level generalized utilitarianism uses a value function that is equal to the sum of differences between transformed individual utilities and the transformed critical level.

A feature that is common to all of the solutions to pure population problems mentioned above (and others - see, for instance, Hurka [1983] and $\mathrm{Ng}$ [1986]) is that they are rationalizable: the solution selects the optimal population-size-per-capita-consumption pair(s) according to some social ordering. ${ }^{7}$ However, pure population problems are, by definition, choice problems, and this raises the question whether there might be other solutions with attractive properties that are left out of consideration by restricting attention to rationalizable solutions.

The rationalizability of a choice function is a problem that has its origins in the literature on consumer theory; see, for instance, Blackorby, Bossert, and Donaldson [1995a], Bossert [1993], Gale [1960], Houthakker [1950], Hurwicz and Richter [1971], Kihlstrom, Mas-Colell, and Sonnenschein [1976], Peters and Wakker [1994], Rose [1958], Samuelson [1938, 1948], and Uzawa [1960, 1971] for discussions of that issue. Extensions to more general choice problems can be found, for example, in Arrow [1959], Baigent and Gaertner [1996], Bossert [1995], Hansson [1968], Richter [1966, 1971], and Sen [1971, 1993]. The rationalizability of various group-decision procedures (such as bargaining solutions) has been analyzed in contributions such as Bossert [1994], Lensberg [1987], and Peters and Wakker [1991].

The purpose of this paper is to examine the rationalizability issue in the context of the pure population problem. It turns out that, due to the structure of pure population problems, efficient solutions are rationalizable if and only if a weak condition regarding the choice of zero as an optimal population size is satisfied. If zero were excluded as a possible population size, all efficient solutions would be rationalizable. However, excluding zero as

\footnotetext{
6 This is true in any population problem. See Blackorby and Donaldson [1984].

7 Usually, social orderings defined on pairs of population sizes and per-capita utilities are employed, assuming that the utility function defined on consumption levels is the same for everybody. Under a weak efficiency requirement which we impose throughout the paper, it does not matter whether per-capita consumption or per-capita utility is used. See Section 3 for a discussion.
} 
a possibility for all amounts of the resource would be an implausible assumption - for very low values of the resource, it seems natural to allow for the possibility that it is best not to have anybody alive.

The natural set of possible population sizes is the set of nonnegative integers. In some economic models, however, it is convenient to treat population size as a continuous variable - any nonnegative real number is feasible. Our main result regarding the rationalizability of solutions to pure population problems is valid for either of these two possibilities. In Section 4, however, a homogeneity property is used, which is only meaningful if the set of possible population sizes consists of the nonnegative real numbers. For discrete population sizes, the condition can only be approximately satisfied due to integer problems.

Section 2 introduces our notation and a formal definition of the pure population problem discussed in this paper. Rationalizability is defined in Section 3, and a necessary and sufficient condition for the rationalizability of solutions to pure population problems is presented. Section 4 discusses rationalizability by critical-level generalized utilitarian social orderings. Section 5 provides some concluding remarks.

\section{Pure Population Problems}

The set of nonnegative integers is denoted by $\mathcal{Z}_{+}$, and the set of all (nonnegative, positive) real numbers is $\mathcal{R}\left(\mathcal{R}_{+}, \mathcal{R}_{++}\right)$. The set of feasible population sizes is denoted by $\mathcal{N}$, where either $\mathcal{N}=\mathcal{Z}_{+}$or $\mathcal{N}=\mathcal{R}_{+}$. Except for Section 4, it does not matter which of those two options is chosen. Let $\mathcal{N}_{++}=\mathcal{N} \backslash\{0\}$.

A pure population problem is defined by the total amount of the resource $\omega \in \mathcal{R}_{++}$ available. Each pure population problem $\omega$ induces a set of feasible population-size-percapita-consumption pairs defined by

$$
B(\omega)=\left\{(n, x) \mid n \in \mathcal{N}_{++} \text {and } 0 \leq x \leq \omega / n\right\} \cup\{(0,0)\} .
$$

The choice of $x=0$ in the case of a zero population is arbitrary and of no consequence for the results of the paper.

Let $\mathcal{P}_{+}=\mathcal{N}_{++} \times \mathcal{R}_{+}$, and let $\mathcal{P}=\mathcal{P}_{+} \cup\{(0,0)\}$. The set of all nonempty subsets of $\mathcal{P}$ is denoted by $2^{\mathcal{P}}$. A solution to the pure population problem is a choice function defined on $\mathcal{R}_{++}$, that is, a function $c: \mathcal{R}_{++} \longrightarrow 2^{\mathcal{P}}$ such that, for all $\omega \in \mathcal{R}_{++}, c(\omega) \subseteq B(\omega)$.

A requirement which we impose on $c$ throughout the paper is a weak efficiency condition. Efficiency requires that the entire amount of the resource available is distributed by a solution.

Efficiency: For all $\omega \in \mathcal{R}_{++}$, for all $n \in \mathcal{N}_{++}$, for all $x, y \in \mathcal{R}_{+}$such that $x>y$, if $(n, x) \in B(\omega)$, then $(n, y) \notin c(\omega)$. 


\section{Rationalizable Solutions}

A solution $c$ is rationalizable if and only if there exists an ordering 8 $\succeq$ on $\mathcal{P}$ such that, for all $\omega \in \mathcal{R}_{++}$,

$$
c(\omega)=\{(n, x) \in B(\omega) \mid(n, x) \succeq(m, y) \text { for all }(m, y) \in B(\omega)\} .
$$

That is, $c$ is rationalizable if and only if there exists an ordering $\succeq$ such that, for each $\omega \in \mathcal{R}_{++}$, the choice function chooses the set of best elements in the feasible set $B(\omega)$ according to $\succeq$. In this case, we say that $\succeq$ rationalizes $c$ or $\succeq$ is a rationalization of $c$. The strict preference relation and the indifference relation corresponding to $\succeq$ are denoted by $\succ$ and $\sim$ respectively.

The efficiency assumption imposed on $c$ implies that rationalizing orderings can be chosen such that they possess the following monotonicity property (see Theorem 1).

Monotonicity: For all $(n, x),(m, y) \in \mathcal{P}$, if $n=m$ and $x>y$, then $(n, x) \succ(m, y)$.

The direct revealed preference relation $R_{c}$ corresponding to the choice function $c$ is defined as follows. For all $(n, x),(m, y) \in \mathcal{P},(n, x) R_{c}(m, y)$ if and only if there exists $\omega \in \mathcal{R}_{++}$such that $(n, x) \in c(\omega)$ and $(m, y) \in B(\omega)$.

The following axiom imposes a restriction on the choice of zero as an optimal population size. Zero consistency requires that if a zero population size is chosen for some amount of the resource, then zero must be the only chosen population size for all lower levels of the resource.

Zero Consistency: If there exists $\bar{\omega} \in \mathcal{R}_{++}$such that $(0,0) \in c(\bar{\omega})$, then $c(\omega)=\{(0,0)\}$ for all $\omega<\bar{\omega}$.

This axiom only imposes a restriction on choices that involve a zero population. Therefore, it is considerably weaker than the usual axioms that are required for rational choice. That this condition is necessary and sufficient for rationalizability is a consequence of the special structure of pure population problems. We obtain

Theorem 1: Let $c$ be an efficient solution. $c$ is rationalizable by a monotonic social ordering if and only if c satisfies zero consistency.

\footnotetext{
8 An ordering is a reflexive, transitive, and complete binary relation.
} 
Proof: (a) Only if: Suppose $c$ is efficient, and let $\succeq$ be a monotonic ordering that rationalizes $c$.

Suppose zero consistency is violated. Then there exist $\bar{\omega}, \omega \in \mathcal{R}_{++}$and $(n, x) \in \mathcal{P}_{+}$ such that $\omega<\bar{\omega},(0,0) \in c(\bar{\omega})$, and $(n, x) \in c(\omega) .(0,0)$ is feasible for any possible amount of the resource, and in particular for $\omega$. Therefore, because $\succeq$ rationalizes $c$, it follows that $(n, x) \succeq(0,0)$. Because $(n, x)$ is feasible for the problem $\omega$, it must be feasible for the higher amount of the resource $\bar{\omega}$. Therefore, because $(0,0)$ is chosen for $\bar{\omega}$ and $(n, x)$ is at least as good as $(0,0)$ according to the rationalizing ordering, it follows that $(n, x) \in c(\bar{\omega})$. But this contradicts efficiency because $n x \leq \omega<\bar{\omega}$.

(b) If: To prove the 'if' part of the theorem, we establish the existence of a monotonic ordering that rationalizes $c$. Suppose $c$ is efficient and satisfies zero consistency. Define the relation $\succeq^{*}$ on $\mathcal{P}$ as follows. For all $(n, x),(m, y) \in \mathcal{P},(n, x) \succeq^{*}(m, y)$ if and only if

$$
\left[\exists t \in \mathcal{R}_{+} \text {such that } x \geq t \text { and }(n, t) R_{c}(m, y)\right] \text { or }[n=m \text { and } x \geq y] .
$$

First, we show that $\succeq^{*}$ is a quasi-ordering. ${ }^{9}$ That $\succeq^{*}$ is reflexive follows immediately. To show that $\succeq^{*}$ is transitive, suppose $(n, x) \succeq^{*}(m, y)$ and $(m, y) \succeq^{*}(r, z)$ for some $(n, x),(m, y),(r, z) \in \mathcal{P}$. We can assume $(n, x) \neq(m, y)$ and $(m, y) \neq(r, z)$ because all other cases are trivial. According to the definition of $\succeq^{*}$, we can distinguish four cases.

1.) $\exists t \in \mathcal{R}_{+}$such that $x \geq t$ and $(n, t) R_{c}(m, y)$, and $\exists s \in \mathcal{R}_{+}$such that $y \geq s$ and $(m, s) R_{c}(r, z)$. The following five subcases arise according to which of the population sizes in these pairs are equal to zero (note that these are the only possibilities because the case $(n, x)=(m, y)$ or $(m, y)=(r, z)$ is ruled out $)$.

(i) $n=0$ and $r=0$. In this case, $(n, x)=(0,0) \succeq^{*}(0,0)=(r, z)$ follows immediately because $\succeq^{*}$ is reflexive.

(ii) $n=0$ and $m \neq 0$ and $r \neq 0$. It follows that $(n, x)=(0,0)$ and, because $x \geq t$, $t=0$. Therefore, $(0,0) R_{c}(m, y)$. Let $\omega, \hat{\omega} \in \mathcal{R}_{++}$be such that $(0,0) \in c(\omega), m y \leq \omega$, $(m, s) \in c(\hat{\omega})$, and $r z \leq \hat{\omega}$. By efficiency, $m s=\hat{\omega}$. Furthermore, $\omega \geq m y \geq m s$ and, therefore, $\omega \geq \hat{\omega}$. If $\omega=\hat{\omega}$, it follows that $(0,0) \in c(\hat{\omega})$ and thus $(n, x)=(0,0) R_{c}(r, z)$, which implies $(n, x) \succeq^{*}(r, z)$. The case $\omega>\hat{\omega}$ cannot occur because it would contradict zero consistency.

(iii) $n \neq 0$ and $m=0$ and $r \neq 0$. Analogously to (ii), $(m, y)=(m, s)=(0,0)$ and hence $(0,0) R_{c}(r, z)$. Let $\omega, \hat{\omega} \in \mathcal{R}_{++}$be such that $(n, t) \in c(\omega),(0,0) \in c(\hat{\omega})$, and $r z \leq \hat{\omega}$. If $\omega<\hat{\omega}$, we obtain a contradiction to zero consistency. Therefore, $\omega \geq \hat{\omega}$, which implies $r z \leq \omega$. Hence, $(r, z) \in B(\omega)$, and it follows that $(n, t) R_{c}(r, z)$ and thus $(n, x) \succeq^{*}(r, z)$.

(iv) $n \neq 0$ and $m \neq 0$ and $r=0$. Therefore, $(r, z)=(0,0)$. Let $\omega \in \mathcal{R}_{++}$be such that $(n, t) \in c(\omega)$. Because $(0,0) \in B(\omega)$, it follows that $(n, t) R_{c}(0,0)=(r, z)$ and hence $(n, x) \succeq^{*}(r, z)$.

\footnotetext{
9 A quasi-ordering is a reflexive and transitive, but not necessarily complete, binary relation.
} 
(v) $n \neq 0$ and $m \neq 0$ and $r \neq 0$. Let $\omega, \hat{\omega} \in \mathcal{R}_{++}$be such that $(n, t) \in c(\omega), m y \leq \omega$, $(m, s) \in c(\hat{\omega})$, and $r z \leq \hat{\omega}$. Efficiency implies $m s=\hat{\omega}$, and we obtain $\omega \geq m y \geq m s=\hat{\omega} \geq$ $r z$. Therefore, $(r, z) \in B(\omega)$, and it follows that $(n, t) R_{c}(r, z)$ and thus $(n, x) \succeq^{*}(r, z)$.

2.) $n=m$ and $x \geq y$, and $m=r$ and $y \geq z$. In this case, we obtain $n=r$ and $x \geq z$ and thus $(n, x) \succeq^{*}(r, z)$.

3.) $\exists t \in \mathcal{R}_{+}$such that $x \geq t$ and $(n, t) R_{c}(m, y)$, and $m=r$ and $y \geq z$. Because $(m, y) \neq(r, z)$, it follows that $m=r \neq 0$. Let $\omega \in \mathcal{R}_{++}$be such that $(n, t) \in c(\omega)$ and $m y \leq \omega$. It follows that $r z=m z \leq m y \leq \omega$ and, therefore, $(r, z) \in B(\omega)$. Hence, $(n, t) R_{c}(r, z)$, and we obtain $(n, x) \succeq^{*}(r, z)$.

4.) $n=m$ and $x \geq y$, and $\exists t \in \mathcal{R}_{+}$such that $y \geq t$ and $(m, t) R_{c}(r, z)$. Therefore, $(n, t) R_{c}(r, z)$. Because $x \geq y \geq t$, this implies $(n, x) \succeq^{*}(r, z)$.

Next, it is shown that $\succeq^{*}$ satisfies monotonicity. Suppose $n=m$ and $x>y$. By definition, $(n, x) \succeq^{*}(n, y)$. Suppose $(n, y) \succeq^{*}(n, x)$. Because $y<x$, it must be the case that there exists $t \in \mathcal{R}_{+}$such that $y \geq t$ and $(n, t) R_{c}(n, x)$. Let $\omega \in \mathcal{R}_{++}$be such that $(n, t) \in c(\omega)$ and $n x \leq \omega$. Note that, because $y \neq x, n \neq 0$. Efficiency implies $n t=\omega$, and we obtain $n y \geq n t=\omega \geq n x$. This implies $y \geq x$, a contradiction. Therefore, $(n, x) \succ^{*}(n, y)=(m, y)$.

The next step is to show that $\succeq^{*}$ rationalizes $c$. Let $\omega \in \mathcal{R}_{++}$and $(n, x) \in c(\omega)$. Therefore, $(n, x) R_{c}(m, y)$ for all $(m, y) \in B(\omega)$ and hence $(n, x) \succeq^{*}(m, y)$ for all $(m, y) \in$ $B(\omega)$. This establishes that

$$
c(\omega) \subseteq\left\{(n, x) \in B(\omega) \mid(n, x) \succeq^{*}(m, y) \text { for all }(m, y) \in B(\omega)\right\} .
$$

To complete the proof that $\succeq^{*}$ rationalizes $c$, it remains to be shown that

$$
\left\{(n, x) \in B(\omega) \mid(n, x) \succeq^{*}(m, y) \text { for all }(m, y) \in B(\omega)\right\} \subseteq c(\omega) .
$$

Suppose $(n, x) \in B(\omega)$ and $(n, x) \succeq^{*}(m, y)$ for all $(m, y) \in B(\omega)$. Let $(r, z) \in c(\omega)$ (note that $c(\omega)$ is, by assumption, nonempty). If $(r, z)=(n, x)$, we are done. Now suppose $(r, z) \neq(n, x)$. Because this excludes the case $n=r=0$, there are three cases left to consider.

1.) $n=0$ and $r \neq 0$. Because $n \neq r,(n, x) \succeq^{*}(r, z)$ implies $(0,0) R_{c}(r, z)$. Let $\hat{\omega} \in \mathcal{R}_{++}$be such that $(0,0) \in c(\hat{\omega})$ and $r z \leq \hat{\omega}$. Efficiency implies $r z=\omega$ and, therefore, $\omega \leq \hat{\omega}$. If $\omega=\hat{\omega},(n, x)=(0,0) \in c(\omega)$ by definition. If $\omega<\hat{\omega},(n, x)=(0,0) \in c(\omega)$ by zero consistency.

2.) $n \neq 0$ and $r=0$. Therefore, $(0,0) \in c(\omega)$. Because $(n, x) \succeq^{*}(r, z)=(0,0)$ and $n \neq 0$, it follows that there exists $t \in \mathcal{R}_{+}$such that $x \geq t$ and $(n, t) R_{c}(0,0)$. Let $\hat{\omega} \in \mathcal{R}_{++}$ be such that $(n, t) \in c(\hat{\omega})$. Zero consistency implies $\hat{\omega} \geq \omega$.

(i) If $\hat{\omega}=\omega$, we obtain $(n, t) \in c(\omega)$, and efficiency implies

$$
n t=\omega .
$$


Furthermore, because $(n, x) \in B(\omega)$, it follows that $n x \leq \omega$. Together with (3.5) and the fact that $x \geq t$, it follows that $x=t$ and hence $(n, x) \in c(\omega)$.

(ii) If $\hat{\omega}>\omega$, efficiency can be used to obtain $n x \geq n t=\hat{\omega}>\omega$, contradicting the feasibility of $(n, x)$ for the problem $\omega$.

3.) $n \neq 0$ and $r \neq 0$. There are two subcases.

(i) $n=r$ and $x \geq y$. It follows that $x>y$ because $(n, x) \neq(r, z)$ by assumption. This contradicts efficiency.

(ii) $\exists t \in \mathcal{R}_{+}$such that $x \geq t$ and $(n, t) R_{c}(r, z)$. Let $\hat{\omega} \in \mathcal{R}_{++}$be such that $(n, t) \in c(\hat{\omega})$ and $r z \leq \hat{\omega}$. Using efficiency, we obtain $n t=\hat{\omega} \geq r z=\omega \geq n x \geq n t$, which implies $\hat{\omega}=\omega$ and $t=x$. Therefore, $(n, x) \in c(\omega)$.

This completes the proof that $\succeq^{*}$ is a monotonic quasi-ordering that rationalizes $c$. Note that $\succeq^{*}$ is not necessarily complete. Therefore, it remains to be shown that $\succeq^{*}$ can be extended to an ordering with the desired properties. Applying Richter's [1966] proof (which uses Szpilrajn's [1930] extension of an antisymmetric quasi-ordering to an antisymmetric ordering), we can find an ordering $\succeq \supseteq \succeq^{*}$ that rationalizes $c$. To see that $\succeq$ satisfies monotonicity, note that Szpilrajn's extension procedure is applied to the antisymmetric relation induced on the equivalence classes of $\succeq^{*}$ and, therefore, all strict preferences in $\succeq^{*}$ that are due to the monotonicity requirement are preserved when extending $\succeq^{*}$ to $\succeq$.

The standard solutions proposed for pure population problems are rationalized by orderings that rank pairs of population sizes and per-capita utilities rather than per-capita consumptions. Given efficiency, this makes no difference. Suppose $U: \mathcal{R}_{+} \longrightarrow \mathcal{R}$ is an increasing utility function defined on individual consumption levels. This function is assumed to be the same for all individuals. By efficiency, only pairs of the form $(n, \omega / n)$ can be selected, and $c$ induces a choice function on pairs of the form $(n, U(\omega / n))$. Conversely, because $U$ is increasing and efficiency is satisfied, $c$ can be recovered uniquely from knowledge of this choice function defined on population-size-per-capita-utility pairs.

\section{Homogeneity and Critical-Level Generalized Utilitarianism}

Suppose $\mathcal{N}=\mathcal{R}_{+}$, that is, population size is treated as a continuous variable. In addition, we restrict attention to solutions that select an interval of population sizes for each value of the resource $\omega$. The following connectedness condition captures this assumption.

Connectedness: For all $\omega \in \mathcal{R}_{++}$, the set $\left\{n \in \mathcal{R}_{+} \mid \exists x \in \mathcal{R}_{+}\right.$such that $\left.(n, x) \in c(\omega)\right\}$ is connected. 
Instead of requiring the above connectedness property with respect to chosen population sizes, we could alternatively impose an analogous property with respect to chosen percapita consumptions. In the presence of efficiency, these two requirements are equivalent, and either of them implies that $c(\omega)$ is a connected set for all $\omega \in \mathcal{R}_{++}$.

In this framework, a plausible property of a solution is homogeneity: if the amount of the resource $\omega$ is multiplied by some positive number $\lambda$ and $n \in \mathcal{R}_{+}$is a chosen population size for $\omega$, then $\lambda n$ is a chosen population size for $\lambda \omega$. This is a reasonable condition because, in a pure population problem, the amount of the resource represents the carrying capacity of the environment.

Homogeneity: For all $\omega, \lambda \in \mathcal{R}_{++}$, for all $n \in \mathcal{R}_{+}$,

$$
\left[\exists x \in \mathcal{R}_{+} \text {such that }(n, x) \in c(\omega)\right] \longleftrightarrow\left[\exists y \in \mathcal{R}_{+} \text {such that }(\lambda n, y) \in c(\lambda \omega)\right] \text {. }
$$

Together with zero consistency, homogeneity can be used to provide a characterization of the critical-level generalized utilitarian solutions to pure population problems, provided that there exists some amount of the resource for which a positive population size is chosen. To define these solutions formally, suppose that each person has the same continuous and increasing utility function $U: \mathcal{R}_{+} \longrightarrow \mathcal{R}$ defined on possible consumption levels. A social ordering $\succeq$ is a critical-level generalized utilitarian ordering if and only if there exist $\alpha \in \mathcal{R}$ and a continuous and increasing function $g: \mathcal{R} \longrightarrow \mathcal{R}$ with $g(0)=0$ such that, for all $(n, x),(m, y) \in \mathcal{P}$

$$
(n, x) \succeq(m, y) \longleftrightarrow n[g(U(x))-g(\alpha)] \geq m[g(U(y))-g(\alpha)] .
$$

The parameter $\alpha$ is the critical level of utility. For $\alpha=0$, we obtain classical generalized utilitarianism. If the function $g$ is (strictly) concave, the resulting ordering represents (strict) inequality aversion in utilities. Because $U$ and $g$ are increasing, these orderings satisfy monotonicity. We obtain

Theorem 2: Let $c$ be an efficient and connected solution, and suppose there exists $\hat{\omega} \in \mathcal{R}_{++}$and $(n, x) \in \mathcal{P}_{+}$such that $(n, x) \in c(\hat{\omega})$. c is rationalizable by a criticallevel generalized utilitarian social ordering if and only if c satisfies zero consistency and homogeneity.

Proof: (a) Only if: Suppose $\succeq$ is a critical-level generalized utilitarian ordering that rationalizes $c$. Theorem 1 implies that zero consistency is satisfied. To show that $c$ is homogeneous, let $\omega, \lambda \in \mathcal{R}_{++}$and $n \in \mathcal{R}_{+}$. It follows that there exists $x \in \mathcal{R}_{+}$such that $(n, x) \in c(\omega)$ if and only if

$$
n[g(U(x))-g(\alpha)] \geq m[g(U(y))-g(\alpha)]
$$


for all $(m, y) \in B(\omega)$. Because $m y \leq \omega$ if and only if $\lambda m y \leq \lambda \omega,(\lambda, y) \in B(\lambda \omega)$ if and only if $(m, y) \in B(\omega)$. Multiplying both sides of (4.3) by $\lambda$, we obtain

$$
\lambda n[g(U(x))-g(\alpha)] \geq \lambda m[g(U(y))-g(\alpha)]
$$

for all $(\lambda m, y) \in B(\lambda \omega)$. Letting $r=\lambda m$, this is equivalent to

$$
\lambda n[g(U(x))-g(\alpha)] \geq r[g(U(y))-g(\alpha)]
$$

for all $(r, y) \in B(\lambda \omega)$ which, in turn, is equivalent to $(\lambda n, x) \in c(\lambda \omega)$.

(b) If: Suppose $c$ is efficient and connected and satisfies zero consistency and homogeneity, and a positive population size is chosen for some value of the resource available. First, we show that we must have

$$
(0,0) \notin c(\omega)
$$

for all $\omega \in \mathcal{R}_{++}$. By way of contradiction, suppose there exists $\omega \in \mathcal{R}_{++}$such that $(0,0) \in c(\omega)$. By assumption, there exists $\hat{\omega} \in \mathcal{R}_{++}$such that $(n, x) \in c(\hat{\omega})$ for some $(n, x) \in \mathcal{P}_{+}$. Choose $m \in \mathcal{N}_{++}$such that $m \hat{\omega}>\omega$. Letting $\lambda=m \hat{\omega} / \omega$, homogeneity implies $(0,0) \in c(m \hat{\omega})$. Because $m \hat{\omega}>\omega$, this contradicts zero consistency.

Our next step is to show that there exists a continuous, increasing, and concave function $\phi: \mathcal{R}_{+} \longrightarrow \mathcal{R}$ such that the ordering defined by

$$
(n, x) \succeq(m, y) \longleftrightarrow n \phi(x) \geq m \phi(y)
$$

for all $(n, x),(m, y) \in \mathcal{P}$ rationalizes $c$. Connectedness implies that the set of chosen population sizes is an interval for each $\omega \in \mathcal{R}_{++}$. Without loss of generality, suppose this interval is closed (it is straightforward to adapt the construction below to cover the other possibilities). Let $\left[n_{L}, n_{H}\right]$ with $n_{L} \leq n_{H}$ be this interval for $\omega=1$. By efficiency, the chosen population-size-per-capita-consumption pairs are of the form $(n, 1 / n)$ for all $n \in\left[n_{L}, n_{H}\right]$. Now define

$$
\phi(x)= \begin{cases}2 x-1 / n_{H} & \text { if } x \in\left[0,1 / n_{H}\right) ; \\ x & \text { if } x \in\left[1 / n_{H}, 1 / n_{L}\right] ; \\ \left(x+1 / n_{L}\right) / 2 & \text { if } x \in\left(1 / n_{L}, \infty\right) .\end{cases}
$$

$\phi$ is continuous, increasing, and concave. Clearly, the ordering defined by (4.7) with the function $\phi$ as in (4.8) rationalizes the choice of the set $\left\{(n, 1 / n) \mid n \in\left[n_{L}, n_{H}\right]\right\}$ from the feasible set $B(1)$. By homogeneity and efficiency, we obtain

$$
c(\omega)=\left\{(\omega n, 1 / n) \mid n \in\left[n_{L}, n_{H}\right]\right\}
$$

for all $\omega \in \mathcal{R}_{++}$, and it follows that $c$ is rationalized by the ordering in (4.7).

Finally, we use $\phi$ to construct a critical level $\alpha$ and a function $g$ as in the definition of critical-level generalized utilitarianism. Let $\bar{x} \in \mathcal{R}_{+}$be such that $\phi(\bar{x})=0$ (such a consumption level $\bar{x}$ exists by definition of $\phi$ ). Because $U$ is continuous and increasing, 
the image of $U$ must be an interval of the form $[a, b)$ with $a \in \mathcal{R}$ and $b \in \mathcal{R}$ or $b=\infty$, and $a<b$. If $b \in \mathcal{R}$, define the function $\bar{g}: \mathcal{R} \longrightarrow \mathcal{R}$ by letting

$$
\bar{g}(y)= \begin{cases}y+\phi\left(U^{-1}(a)\right)-a & \text { if } y \in(-\infty, a) \\ \phi\left(U^{-1}(y)\right) & \text { if } y \in[a, b) \\ y+\phi\left(\lim _{z \rightarrow b} U^{-1}(z)\right)-b & \text { if } y \in[b, \infty)\end{cases}
$$

if $b=\infty, \bar{g}$ is defined by the first two cases in (4.10). Now let $g(y)=\bar{g}(y)-\bar{g}(0)$ for all $y \in \mathcal{R}$. Defining $\alpha=U(\bar{x})$, it follows that

$$
\phi(x)=\phi(x)-\phi(\bar{x})=\bar{g}(U(x))-\bar{g}(U(\bar{x}))=\bar{g}(U(x))-\bar{g}(\alpha)
$$

for all $x \in \mathcal{R}_{+}$and, hence,

$$
\phi(x)=\bar{g}(U(x))-\bar{g}(\alpha)=\bar{g}(U(x))-\bar{g}(0)-\bar{g}(\alpha)+\bar{g}(0)=g(U(x))-g(\alpha)
$$

for all $x \in \mathcal{R}_{+}$. Note that $g$ is continuous and increasing because $U$ and $\phi$ are and, furthermore, $g(0)=0$. Substituting (4.12) into (4.7), it follows that $c$ is rationalized by a critical-level utilitarian social ordering.

Zero consistency and homogeneity are independent. The choice function defined by $c(\omega)=\{(0,0)\} \cup\left\{(n, x) \in \mathcal{P}_{+} \mid n x=\omega\right\}$ for all $\omega \in \mathcal{R}_{++}$satisfies homogeneity but violates zero consistency. If $c(\omega)=\{(1, \omega)\}$ for all $\omega \in \mathcal{R}_{++}$, zero consistency is satisfied but homogeneity is violated. Note that the above examples satisfy efficiency, connectedness, and the requirement that a positive population size is chosen for some $\hat{\omega} \in \mathcal{R}_{++}$.

The assumption that a positive population size is chosen for some amount of the resource is needed in Theorem 2. If there exists $\hat{\omega} \in \mathcal{R}_{++}$such that $(0,0) \in c(\hat{\omega})$, the argument in the proof implies that $c(\omega)=\{(0,0)\}$ for all $\omega \in \mathcal{R}_{++}$. Suppose $g$ and $\alpha$ are such that $c$ is rationalized by the corresponding critical-level generalized utilitarian ordering. It follows that we must have

$$
0[g(U(0))-g(\alpha)]=0>n[g(U(\omega / n))-g(\alpha)]
$$

for all $n, \omega \in \mathcal{R}_{++}$, which requires, because $g$ is increasing, $U(\omega / n)<\alpha$ for all $n, \omega \in \mathcal{R}_{++}$. But this is not possible if the utility function $U$ is not bounded.

Note, however, that a continuous, increasing, and concave function $\phi$ such that the ordering given by (4.7) rationalizes the solution that always selects $(0,0)$ as the only choice does exist. To see this, let $\phi(x)=-1 /(x+1)$ for all $x \in \mathcal{R}_{+}$. But it is not possible to find a function $g$ and a critical level $\alpha$ such that $\phi$ can be written in the form required for critical-level generalized utilitarianism, unless the utility function $U$ is bounded from above. 


\section{Concluding Remarks}

This paper establishes necessary and sufficient conditions for the rationalizability of solutions to pure population problems. Only a weak consistency property regarding the choice of a population size of zero is required to obtain the existence of a rationalizing ordering. If a plausible homogeneity property is added, solutions that are rationalized by critical-level generalized utilitarian social orderings are characterized. This latter result is restricted to solutions that do not always select a population size of zero as the only choice for all values of the resource.

\section{REFERENCES}

Arrow, K., 1959, Rational choice functions and orderings, Economica 26, 121-127.

Baigent, N. and W. Gaertner, 1996, Never choose the uniquely largest: a characterization, Economic Theory 8, 239-249.

Blackorby, C., W. Bossert, and D. Donaldson, 1995a, Multi-valued demand and rational choice in the two-commodity case, Economics Letters 47, 5-10.

Blackorby, C., W. Bossert, and D. Donaldson, 1995b, Intertemporal population ethics: critical-level utilitarian principles, Econometrica 63, 1303-1320.

Blackorby, C., W. Bossert, and D. Donaldson, 1996, Leximin population ethics, Mathematical Social Sciences 31, 115-131.

Blackorby, C., W. Bossert, and D. Donaldson, 1997, Birth-date dependent population ethics: critical-level principles, Journal of Economic Theory, forthcoming.

Blackorby, C. and D. Donaldson, 1979, Numbers and the standard of living: an ethical examination of the pure population problem, Discussion Paper 79-19, University of British Columbia, Department of Economics.

Blackorby, C. and D. Donaldson, 1984, Social criteria for evaluating population change, Journal of Public Economics 25, 13-33.

Bossert, W., 1990a, Maximin welfare orderings with variable population size, Social Choice and Welfare 7, 39-45.

Bossert, W., 1990b, Social evaluation with variable population size: an alternative concept, Mathematical Social Sciences 19, 143-158.

Bossert, W., 1993, Continuous choice functions and the strong axiom of revealed preference, Economic Theory 3, 379-385.

Bossert, W., 1994, Rational choice and two-person bargaining solutions, Journal of Mathematical Economics 23, 549-563.

Bossert, W., 1995, Choices, consequences, and rationality, Working Paper 9501, University of Waterloo, Department of Economics.

Broome, J., 1993, Goodness is reducible to betterness: the evil of death is the value of life, in The Good and the Economical: Ethical Choices in Economics and Management, P. Koslowski, ed., Springer, Berlin, 69-83.

Dasgupta, P., 1993, An Inquiry into Well-Being and Destitution, Clarendon, Oxford. 
Dasgupta, P., 1994, Savings and fertility: ethical issues, Philosophy and Public Affairs 23, 99-127.

Gale, D., 1960, A note on revealed preference, Economica 27, 348-354.

Hansson, B., 1968, Choice structures and preference relations, Synthese 18, 443-458.

Houthakker, H., 1950, Revealed preference and the utility function, Economica 17, 159174.

Hurka, T., 1982, Average utilitarianism, Analysis 42, 65-69.

Hurka, T., 1983, Value and population size, Ethics 93, 496-507.

Hurwicz, L. and M. Richter, 1971, Revealed preference without demand continuity assumptions, in Preferences, Utility, and Demand, J. Chipman, L. Hurwicz, M. Richter, and H. Sonnenschein, eds., Harcourt Brace Jovanovich, New York, 59-76.

Kihlstrom, R., A. Mas-Colell, A., and H. Sonnenschein, 1976, The demand theory of the weak axiom of revealed preference, Econometrica 44, 971-978.

Lensberg, T., 1987, Stability and collective rationality, Econometrica 55, 935-961.

Ng, Y.-K., 1986, Social criteria for evaluating population change: an alternative to the Blackorby-Donaldson criterion, Journal of Public Economics 29, 375-381.

Parfit, D., 1976, On doing the best for our children, in Ethics and Populations, M. Bayles, ed., Schenkman, Cambridge, 100-102.

Parfit, D., 1982, Future generations, further problems, Philosophy and Public Affairs 11, $113-172$.

Parfit, D., 1984, Reasons and Persons, Oxford University Press, Oxford/New York.

Peters, H. and P. Wakker, 1991, Independence of irrelevant alternatives and revealed group preferences, Econometrica 59, 1787-1801.

Peters, H. and P. Wakker, 1994, WARP does not imply SARP for more than two commodities, Journal of Economic Theory 62, 152-160.

Richter, M., 1966, Revealed preference theory, Econometrica 34, 635-645.

Richter, M., 1971, Rational choice, in Preferences, Utility, and Demand, J. Chipman, L. Hurwicz, M. Richter, and H. Sonnenschein, eds., Harcourt Brace Jovanovich, New York, 29-58.

Rose, H., 1958, Consistency of preference: the two-commodity case, Review of Economic Studies 25, 124-125.

Samuelson, P., 1938, A note on the pure theory of consumer's behaviour, Economica 5, 61-71.

Samuelson, P., 1948, Consumption theory in terms of revealed preference, Economica 15, $243-253$.

Sen, A., 1971, Choice functions and revealed preference, Review of Economic Studies 38, 307-317.

Sen, A., 1993, Internal consistency of choice, Econometrica 61, 495-521.

Szpilrajn, E., 1930, Sur l'extension de l'ordre partiel, Fundamenta Mathematicae 16, 386389.

Uzawa, H., 1960, Preference and rational choice in the theory of consumption, in Mathematical Methods in the Social Sciences, 1959, K. Arrow, S. Karlin, and P. Suppes, eds., Stanford University Press, Stanford, 129-148. 
Uzawa, H., 1971, Preference and rational choice in the theory of consumption, in Preferences, Utility, and Demand, J. Chipman, L. Hurwicz, M. Richter, and H. Sonnenschein, eds., Harcourt Brace Jovanovich, New York, 7-28. 